\title{
Editorial: Introduction to the 34th Annual Gallery of Fluid Motion (Portland, Oregon, USA, 2016)
}

\author{
Derek C. Tretheway \\ Portland State University, Portland, Oregon 97201, USA \\ (Received 29 August 2017; published 29 September 2017)
}

DOI: 10.1103/PhysRevFluids.2.090001

The 34th Annual Gallery of Fluid Motion (GFM) was held at the American Physical Society (APS) Division of Fluid Dynamics (DFD) Annual meeting in Portland, Oregon, November 20-22, 2016. The meeting presented approximately 2700 technical abstracts with the GFM contributing an additional 116 entries for display during the meeting (49 posters and 67 video submissions). Submissions were received from 18 countries with approximately $40 \%$ of the submissions from institutions outside the United States. The works presented represent a broad spectrum of fluid mechanics topics conducted in academia, national laboratories, and industry. Overall, the GFM continues to thrive with the number of entries largely tracking the overall size of the APS-DFD meeting [Fig. 1(a)]. With increased computing and imaging resources, video entries to the GFM have increased from an historical norm of approximately $35 \%$ to nearly $60 \%$ [Fig. 1(b)].

To select winners of the 2016 GFM, two panels of judges (six for videos and four for posters) were assembled from eminent scholars with a diverse background in fluid mechanics. As in previous years, judges evaluated entries on the basis of aesthetic appeal, novelty, and creativity and rewarded entries that celebrated the art and beauty of fluids. Poster judges were asked to create a ranked list of the posters. Videos were judged in two stages. First, each judge scored approximately 25 videos with each video being evaluated by three judges. Then, the resulting top 15 videos were ranked by each judge from 1 to 15 . In this manner, six winning posters and six winning videos were identified. For the 7th year, the top three winners in each category were designated the Milton van Dyke Award winners and received a cash honorarium in addition to certificates and copies of An Album of Fluid Motion provided to all winning entries. The winning entries may be viewed online at https://gfm.aps.org. The winning GFM videos and posters are:

\section{Milton van Dyke Award video winners}

Sweeping jet from a fluidic oscillator in crossflow. Florian Ostermann, Philipp Godbersen, Rene Woszidlo, C. Navid Nayeri, and C. Oliver Paschereit.

Shear joy of watching paint dry. R. C. Hurd, N. B. Speirs, J. Belden, Z. Pan, B. Lovett, W. Robinson, M. A. Zamora, S. I. Sharker, M. M. Mansoor, A. Merritt, and T. T. Truscott

Dynamic vortex arrays created by starfish larvae. William Gilpin, Vivek N. Prakash, and Manu Prakash.

\section{Milton van Dyke Award poster winners}

Formation of mini vortex rings arising from a vortex pair impinging on a wavy wall. Sarah E. Morris and C. H. K. Williamson.

Progressive Monte Carlo rendering of atmospheric flow features across scales. Tobias Günther, Alexander Kuhn, Hans-Christian Hege, Markus Gross, and Holger Theisel.

Turbulent horizontal convection at high Schmidt numbers. Pierre-Yves Passaggia, Matthew W. Hurley, Brian White, and Alberto Scotti. 
DEREK C. TRETHEWAY

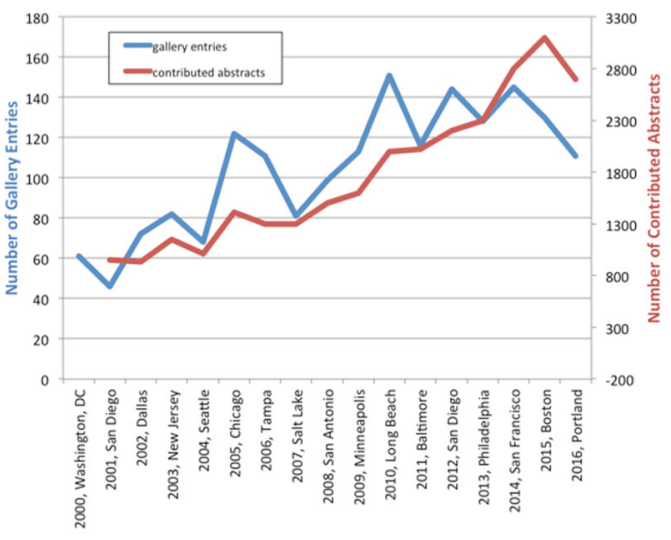

(a)

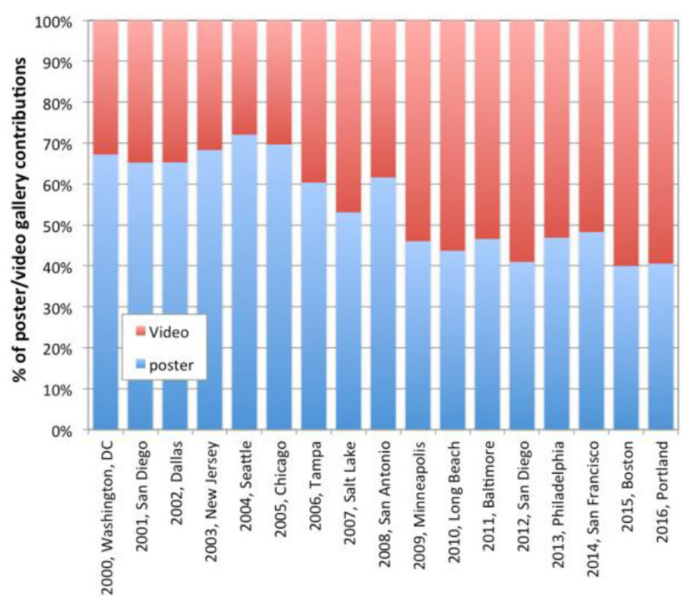

(b)

FIG. 1. (a) Historical growth of contributed abstracts and entries to the Gallery of Fluid Motion. (b) Percentage of poster and video entries to the Gallery of Fluid Motion.

\section{Gallery of Fluid Motion video winners}

Before the bubble ruptures. Li Shen, Fabian Denner, Neal Morgan, Berend van Wachem, and Daniele Dini.

Fall and fragmentation of liquid metal in a viscous fluid. Jean-Baptiste Wacheul and Michael Le Bars.

Flight of a falling maple seed. Injae Lee and Haecheon Choi.

\section{Gallery of Fluid Motion poster winners}

Accelerated condensation in an ultrasonic field. Thomas R. Boziuk, Marc K. Smith, and Ari Glezer. Numerical simulation of liquid round jet atomization. Dorrin Jarrahbashi, William A. Sirignano, Pavel P. Popov, and Fazle Hussain.

Large-eddy simulation of a stratocumulus cloud. Georgios Matheou, Daniel Chung, and João Teixeira.

In conclusion, I would like thank all of the contributors to the 2016 GFM as well the judges for making the 2016 GFM a success. Special thanks to Professor Kenneth Kiger for his continued overall coordination of the GFM and his assistance to the local organizing committee including providing the historical data of Fig. 1. In addition, I would like to thank Paul Dlug and Mark Doyle of APS, Peggy Holland of Meetings and More, and the APS staff for providing logistical support. 